\title{
PRODUCTS OF NEARLY COMPACT SPACES
}

\author{
BY \\ C. T. SCARBOROUGH AND A. H. STONE(1)
}

1. Introduction. Topological properties similar to, but slightly weaker than, compactness have often been considered (see [22, pp. 901, 902] for a partial list) and it would be desirable to know to what extent the analog of Tychonoff's theorem (that every product of compact spaces is compact) will hold for them. That it is liable not to hold in general is shown by J. Novak's example [17], in which the product of two countably compact regular spaces is so far from compact as to have an open-closed infinite discrete subspace. On the other hand, Chevalley and Frink [10] have shown that every product of absolutely closed spaces is absolutely closed; Ikenaga [15] has proved a similar theorem for minimal Hausdorff spaces $\left({ }^{2}\right)$; and Glicksberg [11] has demonstrated that the product theorem holds for pseudocompact spaces under simple supplementary hypotheses (though not without them).

In this paper, we first (\$2) extend the known results about absolutely closed and minimal Hausdorff spaces, reformulating them so that they apply to nonHausdorff spaces. For minimal regular spaces the question is still open; however, we prove some results in this direction in $\S 3$. We obtain partial results for products of feebly compact spaces in $\$ 4$ (our results here are slight generalizations of those of Glicksberg [11]), and for products of sequentially compact spaces in $\$ 5\left(^{(3)}\right.$.

We use the term "space" to mean "topological space", no separation axioms being assumed unless they are stated explicitly. "Compact" thus does not imply "Hausdorff", but coincides with Bourbaki's "quasicompact". We denote the space $(X, \mathscr{T})$ by $X$, leaving the topology $\mathscr{T}$ to be understood. If $\left\{X_{a} \mid a \in A\right\}$ is a family of spaces, we write their product $X=\prod\left\{X_{a} \mid a \in A\right\}$ as $\prod X_{a}$ for short; it is understood that $X$ is given the usual product topology, and (to eliminate

Presented to the Society, January 24, 1964, in part, under the title The product theorem for minimal Hausdorff spaces and related results; received by the editors September 14, 1965.

(1) The first author's contribution is part of a doctoral dissertation at Tulane University. The second author's contribution was supported by a grant from the National Science Foundation, GP 2578.

(2) Also obtained independently by the present authors; see Abstracts 608-107, 64 T-16, in the Notices Amer. Math. Soc. 11 (1964), 107, 130.

(3) Sequential compactness, not being weaker than compactness, may seem an intruder here. The results for sequential compactness were originally formulated for first countable, countably compact spaces; the generalization to sequentially compact spaces gives no more trouble. 
trivial exceptions in stating theorems) that no $X_{a}$ is empty. The projection of $X$ onto $X_{a}$ is denoted by $\pi_{a}$. If $x \in X, \pi_{a}(x)$ is also written as $x_{a}$. If $\mathscr{F}$ is a family of subsets of $X, \pi_{a} \mathscr{F}$ denotes the family $\left\{\pi_{a}(F) \mid F \in \mathscr{F}\right\}$ of subsets of $X_{a}$; thus if $\mathscr{F}$ is a filter base, then so is $\pi_{a} \mathscr{F}$. An open (closed) filter base is a filter base consisting of open (closed) sets. A regular filter base is an open filter base which is equivalent to a closed filter base. The adherence of a filter base $\mathscr{F}$ is denoted by ad $\mathscr{F}$. If $A$ is a subset of a space $X$, the closure of $A$ is written $\bar{A}$ or $\mathrm{Cl}(A)$. Finally, a space $X$ is "regular" if each point of $X$ has a base of closed neighborhoods; the $T_{1}$ axiom is not assumed. A similar remark applies to "completely regular".

2. $H($ i) and $H($ ii) spaces.

2.1. We recall that a Haisdorff space is said to be absolutely closed $\left({ }^{4}\right)$ if it is closed in every Hausdorff space in which it is imbedded, and minimal Hausdorff if it has no strictly smaller (less fine, weaker) Hausdorff topology. As is well known, every compact Hausdorff space is minimal Hausdorff, and every minimal Hausdorff space is absolutely closed, and both converse statements are false - a result which we shall sharpen later. As observed by Bourbaki $[9$, p. 160], absolute closedness is equivalent (for Hausdorff spaces) to the condition:

$H(i)$ Every open filter base on $X$ has nonvoid adherence; and minimal Hausdorffness is equivalent to:

$H$ (ii) Every open filter base on $X$ which has a unique adherent point $x$ is convergent (necessarily to $x$ ) $\left(^{5}\right)$.

We shall study these properties $H(\mathrm{i})$ and $H$ (ii) for spaces which are not necessarily Hausdorff; and for this purpose it is desirable to refine $H(i i)$ further. We say that a space $X$ is (or satisfies) $H$ (ii) nonvacuously if $X$ satisfies $H$ (ii) and moreover has at least one open filter base with unique adherent point. (This additional requirement is certainly met if $X$ is Hausdorff and nonempty; but it can happen more generally, as is shown in Example 2.3 below.) The same reasoning ([3], [5]) which shows that, for Hausdorff spaces, $H$ (ii) implies $H(i)$, also shows more generally that any space which is $H$ (ii) nonvacuously must be $H(\mathrm{i})$. However, a space can satisfy $H($ ii) vacuously without being $H(i)$; see Example 2.2 below.

We remark that $H(\mathrm{i})$ is equivalent to the property that every open covering of $X$ has a finite dense subsystem (property (A) of the list in [22]), and that for regular spaces $H(\mathrm{i})$ is equivalent to compactness.

2.2. EXAMPLE. Let $Q$ be the space of rational numbers, in its usual topology, and let $E$ be a countably infinite set topologized so that its closed proper subsets

(4) Or " $H$-closed" in the terminology of Alexandroff and Hopf [1]; "Hausdorff complete" in the terminology of Banaschewski [3], [4]. We are grateful to M. P. Berri for calling these papers of Banaschewski to our attention.

(5) These properties are called $S($ i) and $S$ (ii) by Berri [5]. We have renamed them so as to have $H$ for Hausdorff, $R$ for regular (see $\S 3$ ). 
are precisely its finite subsets. Then $Q \times E$ is a countable $T_{1}$ space which satisfies $H$ (ii) vacuously but does not satisfy $H(\mathrm{i})$.

2.3. ExAmple. Let $X=E \cup\{p\}$, where $E$ is the space used in the previous example and where $p$ is an extra, isolated point. Then $X$ is a countable $T_{1}$ space which satisfies $H$ (ii) nonvacuously (and hence also satisfies $H(i)$ ), but it is clearly non-Hausdorff.

2.4. THEOREM. If $X=\prod X_{a}$, then $X$ is $H(\mathrm{i})$ if and only if each $X_{a}$ is $H(\mathrm{i})$.

When the spaces are Hausdorff (so that $H$ (i) coincides with absolute closedness), the nontrivial implication here ("if") is proved by Chevalley and Frink in [10, Theorem 3]; and their proof applies here without change.

2.5. LEMMA. Let $X=\prod X_{a}$, where each $X_{a}$ is an $H(\mathrm{i})$ space. If $\mathscr{F}$ is an open filter base on $X$, and if $t \in \operatorname{ad} \pi_{b} \mathscr{F}$ for some $b \in A$, then there exists a point $x \in \operatorname{ad} \mathscr{F}$ such that $x_{b}=t$.

Let $\mathscr{N}$ be the family of open neighborhoods of $t$ in $X_{b}$. Then

$$
\left\{\pi_{b}^{-1}(N) \cap F: N \in \mathscr{N}, F \in \mathscr{F}\right\}
$$

is an open filter base on $X$, and is therefore contained in a maximal open filter base $\mathscr{M}$. We take $x_{b}=t, x_{a} \in$ ad $\pi_{a} \mathscr{M}$ for $a \in A-\{b\}$, and verify (as in the proof of $[10$, Theorem 3$])$ that this works.

2.6. Lemma. If $X \times Y$ satisfies $H$ (ii) nonvacuously, then so does $X$.

Let $\mathscr{F}$ be an open filter base on $X$ with a unique adherent point $x$. By hypothesis, there exists an open filter base $\mathscr{G}$ on $X \times Y$ having a unique adherent point, say $(t, y)$. Since $X \times Y$ is $H$ (ii) nonvacuously, it is $H(\mathrm{i})$, and therefore $X$ and $Y$ are $H(i)$. From the preceding lemma, $\pi_{1} \mathscr{G}$ and $\pi_{2} \mathscr{G}$ have the unique adherent points $t$ and $y$ respectively. Then the open filter base

$$
\mathscr{H}=\left\{F \times \pi_{2} G \mid F \in \mathscr{F}, G \in \mathscr{G}\right\}
$$

has the unique adherent point $(x, y)$. Since $X$ is $H(i i), \mathscr{H}$ converges to $(x, y)$, whence $\mathscr{F}$ converges to $x$. Thus $X$ satisfies condition $H(\mathrm{ii})$; and it must do so nonvacuously because $\pi_{1} \mathscr{G}$ has the unique adherent point $t$.

2.7. THEOREM. If $X=\prod X_{a}$, then $X$ is $H$ (ii) nonvacuously if and only if each $X_{a}$ is $H($ ii) nonvacuously.

"Only if" is immediate from the preceding lemma, so we assume that each $X_{a}$ is $H$ (ii) nonvacuously; it is then $H$ (i) also. Suppose $\mathscr{F}$ is an open filter base on $X$ with a unique adherent point $x$. Then, for each $a \in A, x_{a}$ is an adherent point of $\pi_{a} \mathscr{F}$, and by Lemma 2.5 it must be the only one. Hence $\pi_{a} \mathscr{F}$ converges to $x_{a}$ for each $a \in A$, whence $\mathscr{F}$ converges to $x$, and $X$ is $H($ ii). 
By hypothesis, for each $a \in A$ there exists an open filter base $\mathscr{F}_{a}$ on $X_{a}$ having a unique adherent point $t_{a}$. The product filter base $\prod \mathscr{F}_{a}$ (see $[9$, pp. 69, 70]) will then have the unique adherent point $t$. Thus $X$ is $H$ (ii) nonvacuously.

2.8. Corollary (IKenaga). If $X=\prod X_{a}$, then $X$ is minimal Hausdorff if and only if each $X_{a}$ is minimal Hausdorff $\left(\operatorname{see}\left({ }^{2}\right)\right)$.

This is the special case of the theorem in which all the spaces are Hausdorff.

2.9. THEOREM. If $X=\prod X_{a}$, then $X$ satisfies $H$ (ii) vacuously if and only if at least one $X_{a}$ does so( $\left.{ }^{6}\right)$.

Suppose some $X_{a}$, say $X_{1}$, is $H$ (ii) vacuously, and write $X_{2}$ for the product of all the others. Let $\mathscr{F}$ be a maximal open filter base on $X=X_{1} \times X_{2}$, with an adherent point $\left(x_{1}, x_{2}\right)$. Then $\pi_{1} \mathscr{F}$ is a maximal open filter base on $X_{1}$ (see [18, p. 3]), and $x_{1}$ is an adherent point of $\pi_{1} \mathscr{F}$. Since $X_{1}$ is $H(i i)$ vacuously, $\pi_{1} \mathscr{F}$ must have another adherent point, say $t_{1}$. By maximality, $\pi_{1} \mathscr{F}$ converges to $t_{1}$ also; and similarly $\pi_{2} \mathscr{F}$ converges to $x_{2}$. Hence $\mathscr{F}$ converges to both $\left(x_{1}, x_{2}\right)$ and $\left(t_{1}, x_{2}\right)$. Thus no open filter base on $X$ has a unique adherent point.

Conversely, if each $X_{a}$ has an open filter base $\mathscr{F}_{a}$ with a unique adherent point $x_{a}$, then the product filter base $\prod_{\mathscr{F}_{a}}$ will have $x=\prod x_{a}$ as its unique adherent point.

2.10. Corollary. If $X=\prod X_{a}$ and each $X_{a}$ is $H$ (ii), then so is $X$.

Note that the converse of this corollary, as it stands, is false, even for a product of two factors. The space $Q \times E$ of Example 2.2 satisfies $H$ (ii) (vacuously), but $Q$ does not.

2.11. THEOREM. There exist minimal Hausdorff spaces of arbitrary infinite cardinality which are not compact.

We begin with a standard example, due to Urysohn [24], of a countably infinite space $X$ which is minimal Hausdorff but not compact; see [5, p. 98] for details. Given an infinite cardinal $K$, take $Z$ to be any compact Hausdorff space of $K$ points - for instance, the 1-point compactification of a discrete space of $K$ points. The space $X \times Z$ is of cardinality $K$; it is minimal Hausdorff by Corollary 2.8; and it is noncompact because $X$ is noncompact $\left({ }^{7}\right)$.

2.12. CoRollary. For each cardinal $K \geqq c$, there exists a minimal Hausdorff space of cardinality $K$ which is nowhere locally compact.

For, with the preceding notation, $X^{x_{0}} \times Z$ is such a space. Note that this

(6) We assume in the proof that the set $A$ has at least 2 elements; the theorem is trivial in the remaining cases.

(7) We could of course use the "sum" (disjoint union) of $X$ and $Z$ instead of their product; but the proof, though more elementary, would be longer. 
corollary does not hold when $K=\aleph_{0}$; in fact, by a theorem of Berri [6, Theorem 4], every countable minimal Hausdorff space has an isolated point.

2.13. THEOREM. There exist absolutely closed spaces of arbitrary infinite cardinality which are not minimal Hausdorff.

The proof is similar to that of Theorem 2.11 ; we replace $X$ by a suitable subspace $Y$ (consisting of the points $a_{i j}, c_{i}, a$, where $i, j=1,2, \cdots$, in the notation of [5]) which is absolutely closed but not minimal Hausdorff. The space $Y \times Z$ is absolutely closed by Theorem 2.4, and is not minimal Hausdorff by Corollary 2.8 .

2.14. It has been shown by M. H. Stone [23, p. 435] (see also Obreanu [21] for a simpler proof) that every Hausdorff space can be embedded as a dense subset of some absolutely closed space. Analogously, Berri has asked [5, p. 105] whether every Hausdorff space $X$ can be embedded in some minimal Hausdorff space. If $X$ is completely regular, the answer is of course "yes", since $X$ can then be embedded in a compact Hausdorff space (e.g. $\beta X)$. We give another sufficient condition, but the general question remains open $\left(^{8}\right)$. First, a definition: Given a family $\left\{\mathscr{T}_{a} \mid a \in A\right\}$ of topologies on a set $X$, the smallest (coarsest, weakest) topology on $X$ which contains all the topologies $\mathscr{T}_{a}$ is said to be "generated" by the family $\left\{\mathscr{T}_{a} \mid a \in A\right\}$. It is the topology having $\bigcup\left\{\mathscr{T}_{a} \mid a \in A\right\}$ as subbase.

2.15. THEOREM. If a space $X$ has its topology generated by a family of topologies, each of which is embeddable in a minimal Hausdorff space, then $X$ can be embedded in a minimal Hausdorff space.

Suppose the topology $\mathscr{T}$ of $X$ is generated by the family $\left\{\mathscr{T}_{a} \mid a \in A\right\}$ of topologies, and write $X_{a}$ for $X$ with topology $\mathscr{T}_{a}$. One can verify that the diagonal map $f$ of $X$ in $\prod X_{a}$ (that is, $f(x)_{a}=x$ for all $a \in A$ ) is a homeomorphism. By Corollary 2.8 , if each $X_{a}$ is embeddable in a minimal Hausdorff space $Y_{a}$, this embeds $X$ in the minimal Hausdorff space $\prod Y_{a}$.

2.16. COROLLARY. If the topology of $X$ is generated by a family of minimal Hausdorff topologies, then $X$ can be embedded in a minimal Hausdorff space.

We remark that the converse of Theorem 2.15 is trivially true (through of course this theorem does not solve the problem); however, the converse of Corollary 2.16 is not true. For if $X$ is a countable Hausdorff space without isolated points, it follows from the theorem of Berri quoted in 2.12 that the topology $\mathscr{T}$ of $X$ contains no minimal Hausdorff topologies, and so $\mathscr{T}$ is not generated by such topologies. Such an $X$ can be completely regular, and is then embeddable

(8) For dense embedding, the question has been answered by Banaschewski [4]: a Hausdorff space $X$ can be densely embedded in some minimal Hausdorff space if, and only if, $X$ is semiregular. 
in a minimal Hausdorff space. On the other hand, such an $X$ need not be regular (see [12, p. 65] and [13, p. 508] for examples); and it would be interesting to know it if can then be embedded in a minimal Hausdorff space.

It has recently been shown $\left({ }^{9}\right)$ that every Hausdorff space can be embedded as a closed subset of an absolutely closed space, and every space $X$ can be embedded in a compact (not necessarily Hausdorff) space $X^{*}$ (for instance, its 1-point compactification), and $X^{*}$ will be both $H(\mathrm{i})$ and $H(\mathrm{ii})$-even nonvacuously if we add an extra isolated point to $X^{*}$; moreover, $X^{*}$ will be $T_{1}$ if $X$ is.

The following result will be useful later.

2.17. THEOREM. Let $X$ be an H(i) space, and $Y$ an arbitrary space. If $A$ is an open subset of $X \times Y$, then $\pi_{2}(\bar{A})$ is closed.

Let $y$ be any point of $\mathrm{Cl}\left(\pi_{2}(\bar{A})\right)$; we prove $y \in \pi_{2}(\bar{A})$. Let $\mathscr{N}$ be the open neighborhood system of $y$; then $\mathscr{F}=\{(X \times N) \cap A \mid N \in \mathscr{N}\}$ is an open filter base on $X \times Y$, so $\pi_{1} \mathscr{F}$ has nonvoid adherence. Take $x \in \operatorname{ad} \pi_{1} \mathscr{F}$; then, for every neighborhood $V$ of $x$ and for all $N \in \mathscr{N},[(X \times N) \cap A] \cap(V \times Y) \neq \varnothing$. Thus $(x, y) \in \bar{A}$, and $y \in \pi_{2}(\bar{A})$.

In particular, this theorem applies whenever $X$ is absolutely closed.

3. $R(\mathrm{i})$ and $R(\mathrm{ii})$ spaces.

3.1. A regular $T_{1}$ space will be called " $R$-closed" if it is closed in every regular $T_{1}$ space in which it is embedded; it is said to be "minimal regular" if it has no strictly smaller regular $T_{1}$ topology. Banaschewski [3], and Berri and Sorgenfrey [7], have shown that, for regular $T_{1}$ spaces, $R$-closedness is equivalent to the condition:

$R(i)$ Every regular filter base has nonvoid adherence; and minimal regularity is equivalent to:

$R$ (ii) Every regular filter base which has a unique adherent point is convergent.

Again we shall consider these properties for spaces which need not satisfy any separation axioms. We say that a space $X$ satisfies (or is) $R$ (ii) nonvacuously if it satisfies $R$ (ii) and moreover there exists a regular filter base on $X$ which has a unique adherent point. (This additional requirement is certainly met if $X$ is regular $T_{1}$ and nonempty; but it can happen more generally.) The same reasoning [7, p. 454] which shows that, for regular $T_{1}$ spaces, $R($ ii) implies $R(i)$, also shows more generally that any space which is $R$ (ii) nonvacuously must be $R(\mathrm{i})$. However, a space can be $R$ (ii) vacuously without being $R(\mathrm{i})$; see Examples 2.2 and 3.12 . We shall later answer a question raised in [7] by showing that, even for regular $T_{1}$ spaces, $R($ i) does not imply $R$ (ii). (See Theorem 3.18.)(10)

For regular $T_{1}$ spaces we clearly have compact $\Leftrightarrow$ minimal Hausdorff $\Rightarrow$ minimal regular $\Rightarrow R$-closed; neither of the last two implications is reversible $([7], 3.18)$.

(9) Chen-tung Liu, unpublished.

(10) This question has also been answered independently by Herrlich [14]. 
We also have $H(\mathrm{i}) \Rightarrow R(\mathrm{i})$, and $R$-closed $\Rightarrow$ feebly compact( $\left.{ }^{11}\right)$. For completely regular $T_{1}$ spaces, $R$-closedness is equivalent to compactness [7, Theorem 3]; and this result extends to non- $T_{1}$ spaces, though a different proof is required.

3.2. THEOREM. Every completely regular $R(\mathrm{i})$ space is compact.

Let $\mathscr{F}$ be a filter on a completely regular $R(\mathrm{i})$ space $X$. There exist regular filter bases which are subsets of $\mathscr{F}$ (e.g. $\{X\}$ ), and hence (Zorn's lemma) there exists a maximal such, say $\mathscr{G}$. Since $X$ is $R(\mathrm{i})$, we can take $x \in \mathrm{ad} \mathscr{G}$; we prove $x \in \operatorname{ad} \mathscr{F}$. For if not, then there exists $F \in \mathscr{F}$ such that $x \notin F$; and by complete regularity there exists a real-valued continuous function $f$ on $X$ such that $f(x)=0$ and $f(\bar{F})=1$. For each positive real $a<1$, put $H_{a}=\{x \mid f(x)>a\}$; note that the sets $H_{a}$ form a regular filter base, and that $H_{a} \supset F$. Then

$$
\mathscr{K}=\mathscr{G} \cup\left\{H_{a} \cap G \mid 0<a<1, G \in \mathscr{G}\right\}
$$

is a regular filter base contained in $\mathscr{F}$. By maximality of $\mathscr{G}, \mathscr{K}=\mathscr{G}$, and therefore $H_{a} \in \mathscr{G}$; but $x \notin \mathrm{Cl}\left(H_{a}\right)$, so $x \notin \operatorname{ad} \mathscr{G}$, a contradiction. Therefore $x \in \operatorname{ad} \mathscr{F}$, and $X$ is compact.

3.3. COROLlaRy. If $\mathscr{F}$ is a filter on a completely regular $R(\mathrm{i})$ space, and $\mathscr{G}$ is a regular filter base contained in $\mathscr{F}$ and maximal with respect to these properties, then ad $\mathscr{G}=$ ad $\mathscr{F}$.

This is shown by the proof of 3.2.

3.4. COROllary. Every regular Lindelöf $R(\mathrm{i})$ space is compact.

For it is normal $[16$, p. 113] and hence completely regular; so Theorem 3.2 applies.

3.5. COROLlARY. Every countable regular $R(\mathrm{i})$ space is compact.

3.6. THEOREM. Every continuous image of an $R(\mathrm{i})$ space is $R(\mathrm{i})$.

Proof. Trivial.

3.7. Corollary. If $X=\prod X_{a}$ is $R(\mathrm{i})$, then so is each $X_{a}$.

In particular, if $X$ is $R$-closed, then so is each $X_{a}$. It is unknown whether the converse of this corollary is true, even in the regular $T_{1}$ case. We shall obtain a partial result in this direction later (3.14).

3.8. Theorem. If $X=\prod X_{a}$, then $X$ satisfies $R(\mathrm{ii})$ vacuously if and only if at least one $X_{a}$ does so $(\mathrm{see})\left({ }^{6}\right)$.

(11) For the definition of feeble compactness, see 4.1. In terms of the list in [22], it can be shown that $R(\mathrm{i})$ implies (E), but that even $R$ (ii) nonvacuously does not imply (D) in general. For regular spaces, (D) and (E) coincide with (B) (feeble compactness). 
Suppose some $X_{a}$, say $X_{1}$, is $R$ (ii) vacuously, and write $X_{2}$ for the product of all the others. Let $\mathscr{F}$ be a regular filter base on $X=X_{1} \times X_{2}$, with unique adherent point $\left(a_{1}, a_{2}\right)$; we derive a contradiction. For each $F \in \mathscr{F}$, let $F_{1}$ denote its "section" $\left\{x \mid\left(x, a_{2}\right) \in F\right\}$; we observe that $a_{1} \in F_{1}$, because there exists $F^{\prime} \in \mathscr{F}$ such that $\mathrm{Cl}\left(F^{\prime}\right) \subset F$, and we have $\left(a_{1}, a_{2}\right) \in \mathrm{Cl}\left(F^{\prime}\right) \subset F$. It follows easily that $\left\{F_{1} \mid F \in \mathscr{F}\right\}$ is a regular filter base on $X_{1}$, with unique adherent point $a_{1}$; but this contradicts the assumption on $X_{1}$.

The converse is proved in the same way as in the $H$ (ii) case (2.9); we have only to note that a product of regular filter bases is a regular filter base.

3.9. Theorem. If $X=\prod X_{a}$, and if $X$ is $R$ (ii) nonvacuously, then each $X_{a}$ is $R($ ii) nonvacuously.

As in the proof of the preceding theorem, we may assume that there are only two factors, $X=X_{1} \times X_{2}$. Assuming that $X$ is $R$ (ii) nonvacuously, we deduce that $X_{1}$ has the same property. For if not, then either $X_{1}$ satisfies $R(i i)$ vacuously or it does not satisfy $R$ (ii) at all. The first of these alternatives is excluded here, by Theorem 3.8; hence $X_{1}$ has a regular filter base $\mathscr{F}_{1}$ with a unique adherent point $x_{1}$ to which it does not converge. Also $X_{2}$ has a regular filter base $\mathscr{F}_{2}$ with unique adherent point $x_{2}$, because of Theorem 3.8. The product filter base $\mathscr{F}_{1} \times \mathscr{F}_{2}$ is regular, has $\left(x_{1}, x_{2}\right)$ as its unique adherent point, and does not converge to this point, because $\mathscr{F}_{1}$ does not converge to $x_{1}$. This is a contradiction.

In particular, if a product of (regular $T_{1}$ ) spaces is minimal regular, then so is each factor( $\left.{ }^{12}\right)$.

Again, it is unknown whether the converse of Theorem 3.9 is true, even in the regular $T_{1}$ case (when it is a problem of Berri and Sorgenfrey [7]). We obtain a partial result later (3.16).

For regular (not necessarily $T_{1}$ ) spaces, the property of being $R(\mathrm{ii})$ vacuously can be simplified:

3.10. THEOREM. If $X$ is regular, then $X$ is $R$ (ii) vacuously if and only if, for each $x \in X$, there exists $y \in X-\{x\}$ such that $x$ and $y$ have the same neighborhood systems.

For if $X$ satisfies this last condition, then no filter base has a unique adherent point. Conversely, the neighborhood system of $x \in X$ has at least the adherent point $x$; if there is another adherent point $y$, then every neighborhood of $y$ meets every neighborhood of $x$, from which (in view of regularity) the condition follows.

3.11. CoROllary. If $X$ is regular, then $X$ is $R(\mathrm{ii})$ vacuously if and only if it H(ii) vacuously.

Thus, in the regular case, Theorem 3.8 follows from Theorem 2.9.

(12) This is an unpublished result of M. P. Berri; cf. also [15]. 
From Theorem 3.10 (or 3.8) it is easy to construct a regular space which is $R$ (ii) vacuously without being $R(\mathrm{i})$. (Of course it cannot be $T_{1}$ too.)

3.12. EXAmple. Let $X$ be an infinite discrete space. Let $Y$ be a 2-point space with the trivial topology in which only $\varnothing$ and $Y$ are open. Then $X \times Y$ is regular, and is $R$ (ii) vacuously (from 3.8 or 3.10 ), but it is not $R(\mathrm{i})$. This example also illustrates that $X \times Y$ can be $R$ (ii) vacuously though $X$ is not $R($ ii).

3.13. Lemma. Let $X$ be an $H(\mathrm{i})$ space, $Y$ an $R(\mathrm{i})$ space, and $\mathscr{F}$ a regular filter base on $X \times Y$. Then (i) ad $\pi_{2} \mathscr{F} \neq \varnothing$, (ii) if $y \in \operatorname{ad} \pi_{2} \mathscr{F}$, there exists $x \in X$ such that $(x, y) \in$ ad $\mathscr{F}$.

By Theorem $2.17, \pi_{2} \mathscr{F}$ is a regular filter base on $Y$, so ad $\pi_{2} \mathscr{F} \neq \varnothing$. Let $y \in \operatorname{ad} \pi_{2} \mathscr{F}$, and let $\mathscr{N}$ be the open neighborhood system of $y$. Then

$$
\mathscr{H}=\{(X \times N) \cap F \mid N \in \mathscr{N}, F \in \mathscr{F}\}
$$

is an open filter base on $X \times Y$, and $\pi_{1} \mathscr{H}$ is an open filter base on $X$. Since $X$ is $H(\mathrm{i})$, there exists $x \in \operatorname{ad} \pi_{1} \mathscr{H}$; and $(x, y)$ is easily seen to be an adherent point of $\mathscr{F}$.

We remark that ad $\pi_{1} \mathscr{F} \neq \varnothing$ also; but, to obtain the symmetric situation that to each $x \in \operatorname{ad} \pi_{1} \mathscr{F}$ there corresponds $y \in Y$ such that $(x, y) \in \operatorname{ad} \mathscr{F}$, it seems to be necessary (it is certainly sufficient) to assume $X$ regular and therefore compact.

3.14. Theorem. If $X$ is $H(\mathrm{i})$ and $Y$ is $R(\mathrm{i})$, then $X \times Y$ is $R(\mathrm{i})$.

The proof is immediate from Lemma 3.13.

3.15. COROLLARY The product of a compact Hausdorff(or, equivalently, a regular absolutely closed) space and an $R$-closed space is $R$-closed.

3.16. THEOREM. If $X$ is $H(\mathrm{ii})$ and $Y$ is $R(\mathrm{ii})$, then $X \times Y$ is $R(\mathrm{ii})$.

Let $\mathscr{F}$ be a regular filter base on $X \times Y$ with unique adherent point $(x, y)$. By Lemma 3.13, $y$ is the unique adherent point of $\pi_{2} \mathscr{F}$; also (Theorem 2.17) $\pi_{2} \mathscr{F}$ is a regular filter base on $Y$; hence $\pi_{2} \mathscr{F}$ converges to $y$. Thus, if $x^{\prime} \in \operatorname{ad} \pi_{1} \mathscr{F}$, it readily follows that $\left(x^{\prime}, y\right) \in \operatorname{ad} \mathscr{F}$, and therefore $x^{\prime}=x$. So the open filter base $\pi_{1} \mathscr{F}$ has $x$ as its unique adherent point, and it must converge to $x$. It follows that $\mathscr{F}$ converges to $(x, y)$, as required.

3.17. Corollary (IKenaga [15]). The product of a compact Hausdorff space and a minimal regular space is minimal regular.

3.18. THEOREM. There exist $R$-closed spaces of every uncountably infinite cardinality which are not minimal regular.

As in 2.11 and 2.13 , it will suffice to exhibit an $R$-closed space $T$ of cardinality $\aleph_{1}$ which is not minimal regular; for then the space $X \times T$, where $X$ is a compact 
Hausdorff space of the appropriate cardinality, has the desired properties because of Theorem 3.9 and 3.14. Using the notation of [7, p. 456], we let

$$
T=\bigcup\left\{Z_{i} \mid i \geqq 1\right\} \cup\{p\},
$$

with the induced topology from $Z$, and verify that $T$ is $R$-closed but not minimal regular; a detailed proof is given by Herrlich [14].

The cardinal $\aleph_{0}$ must be excluded in this theorem, because all countable $R$-closed spaces are compact (Corollary 3.5) $\left({ }^{13}\right)$. However, it is not hard to see that there exist $T_{1}$ spaces of cardinal $\aleph_{0}$ which are $R(\mathrm{i})$ but not $R(\mathrm{ii})$.

3.19. THEOREM. There exist minimal regular spaces of every uncountably infinite cardinality which are not compact.

As shown in [7, p. 456], Tychonoff's regular but not completely regular space is in fact minimal regular; and it has cardinal $\aleph_{1}$ and is not compact. (Hence it is not minimal Hausdorff or absolutely closed either.) As in 3.18 we can increase the cardinal as desired. As before, the cardinal $\aleph_{0}$ must be excluded.

The next two results are partial answers to a question raised by Berri: Can every regular space be embedded in a minimal regular space?

3.20. THEOREM. If a space $X$ has its topology generated by an $H(\mathrm{i})(H(\mathrm{ii}))$ topology and an $R(\mathrm{i})(R(\mathrm{ii}))$ topology, then $X$ can be embedded in an $R(\mathrm{i})(R(\mathrm{ii}))$ space.

The proof is similar to that of Theorem 2.15.

3.21. Corollary. If a space $X$ has its topology generated by a compact Hausdorff topology and an R-closed (minimal regular) topology, then $X$ can be embedded in an $R$-closed (minimal regular) space.

As with 2.16, there are spaces-for instance, any countable regular space having no isolated points-to which this corollary does not apply, and for which the question remains open. However, Herrlich [14] has given an example of a regular space which cannot be embedded densely in any $R$-closed space.

\section{Feebly compact spaces.}

4.1. Definition. A space $X$ is said to be "feebly compact", or "lightly compact", if every locally finite system $\mathscr{G}$ of nonempty open sets in $X$ is necessarily finite. This is property $\left(B_{1}\right)$ of the list in $[22$, p. 901$]$, which mentions other characterizations; in particular, $\mathscr{G}$ may be restricted to consist of pairwise disjoint sets. Every $H(\mathrm{i})$ space is feebly compact (because (A) implies (B) in the notation of [22]), and so is every countably compact space; moreover, every feebly compact space is pseudocompact (that is, every continuous real function on the space is bounded). The converses are false, in general [22, p. 902]. But for completely

(13) This remark also shows that Theorem 5 of [6] reduces to Theorem 4 of the same paper. 
regular spaces it is known that pseudocompactness is equivalent to feeble compactness, and for normal $T_{1}$ spaces both these properties coincide with countable compactness (though not with $H(\mathrm{i})$ ).

4.2. THeOREM. If $X=\prod X_{a}$, and $X$ is feebly compact, then so is each $X_{a}$.

The proof is trivial. The question of interest is to what extent the converse is true. Novak's example [17] shows that some supplementary conditions are needed, even for products of 2 factors. We present some sufficient conditions, which together give a slight generalization of a theorem of Glicksberg [11, Theorem 4$]\left({ }^{14}\right)$.

4.3. Definition. We recall that a point $x$ of a space $X$ is said to be a $P$-point (in $X$ ) providing $x$ is interior to every intersection of countably many neighborhoods of $x$. (Examples abound in spaces of ordinals.)

4.4. THEOREM. If $X=\prod X_{a}$, where each $X_{a}$ is feebly compact, and if (except perhaps for one value of a) each non-P point of $X_{a}$ has a countable base of neighborhoods in $X_{a}$, then $X$ is feebly compact.

This is essentially Theorem $4 \mathrm{~b}$ of [11], to which it reduces when the spaces are completely regular (in view of the fact that in a regular feebly compact space each $G_{\delta}$ point has a countable base of neighborhoods, by the same argument as in $[11$, p. 378]). The proof is omitted, as it is substantially the same as Glicksberg's.

We remark that Theorem 6 of [2] is an immediate corollary of the above theorem.

4.5. Lemma. If $X$ is feebly compact and $Y$ is compact then $X \times Y$ is feebly compact.

This is probably known; the proof is straightforward, by familiar technique.

4.6. THEOREM. If $X=\prod X_{a}$, where each $X_{a}$ is feebly compact, and if (except for perhaps one value of a) each $X_{a}$ is locally compact, then $X$ is feebly compact $\left.{ }^{15}\right)$.

This is a slight extension of [11, Theorem 4a], and the idea of the proof is the same; however, the proof in [11] now needs modification, so we give the argument. As in [11] it suffices to prove the theorem for a product of countably many factors, say $X=\prod\left\{X_{n} \mid n=0,1, \cdots\right\}$, where $X_{n}$ is feebly compact for all $n$, and locally compact for $n>0$. Given a system $\mathscr{G}=\{G(m) \mid m=1,2, \cdots\}$ of nonempty open sets in $X$, we must produce a cluster point of $\mathscr{G}$ in $X$. We may assume that each $G(m)$ is an "elementary" set of the form $G(m)=\prod G_{n}(m)$, where $G_{n}(m)$

(14) Glicksberg's results are formulated for completely regular pseudocompact spaces. Thus, in effect, our generalization consists in dropping the requirement of complete regularity.

(15) Here a space is said to be "locally compact" if each point has a compact neighborhood (not necessarily open-or closed); the Hausdorff separation axiom is not required. 
is a nonempty open subset of $X_{n}(n=0,1, \cdots)$. (It will also be true that $G_{n}(m)=X_{n}$ for all $n>n(m)$, but this fact plays no part in the argument.) The system $\left\{G_{1}(m) \mid m=1,2, \cdots\right\}$ of nonempty open subsets of $X_{1}$ has a cluster point $x_{1} \in X_{1}$; let $Y_{1}$ be a compact neighborhood of $x_{1}$; the set $M_{1}=\left\{m \mid Y_{1}\right.$ meets $\left.G_{1}(m)\right\}$ is thus infinite. Recursively we obtain, for each $n=1,2, \cdots$, a compact set $Y_{n} \subset X_{n}$ such that the set $M_{n}=\left\{m \mid m \in M_{n-1}\right.$ and $Y_{n}$ meets $\left.G_{n}(m)\right\}$ is infinite. Let $m_{r}=r$ th member of $M_{r}(r=1,2, \cdots)$; note that, because $M_{1} \supset M_{2} \supset \cdots$, we have $m_{1}<m_{2}<\cdots$, and $m_{r} \in M_{s}$ whenever $r \geqq s$. Consider the space $X_{0} \times Y$, where $Y=\prod\left\{Y_{n} \mid n=1,2, \cdots\right\} ; Y$ is compact, so (by Lemma 4.5 ) $X_{0} \times Y$ is feebly compact. Define, for $r=1,2, \cdots$,

$$
H(r)=G_{0}\left(m_{r}\right) \times\left(G_{1}\left(m_{r}\right) \cap Y_{1}\right) \times \cdots \times\left(G_{r}\left(m_{r}\right) \cap Y_{r}\right) \times Y_{r+1} \times Y_{r+2} \times \cdots,
$$

a nonempty open subset of $X_{0} \times Y$. The system $\{H(r) \mid r=1,2, \cdots\}$ has a cluster point $p$ in $X_{0} \times Y \subset X$. Each neighborhood of $p$ in $X$ contains one of the form $V=\prod\left\{V_{n} \mid n=0,1, \cdots\right\}$, where $V_{n}$ is a neighborhood of $p_{n}$ in $X_{n}$, and where $V_{n}=X_{n}$ if $n>k$. Then the set

$$
V_{0} \times\left(V_{1} \cap Y_{1}\right) \times \cdots \times\left(V_{k} \cap Y_{k}\right) \times Y_{k+1} \times Y_{k+2} \times \cdots
$$

is a neighborhood of $p$ in $X_{0} \times Y$, and it therefore meets $H(r)$ for infinitely many values of $r$; but for each such $r>k$ this means that $V$ meets $G\left(m_{r}\right)$, and hence $p$ is a cluster point of $\mathscr{G}$, as required.

A third result of Glicksberg [11, Theorem 4c] can be extended similarly.

4.7. THEOREM. If $X=\prod X_{a}$, where each $X_{a}$ is feebly compact, and if, for some infinite cardinal $K$, each $X_{a}$ is $K$-compact, and each non-P point of $X_{a}$ has a neighborhood basis consisting of at most $K$ sets, then $X$ is feebly compact.

As in [11] and 4.6, it suffices to prove the theorem for a countable product $X=\prod\left\{X_{n} \mid n=1,2, \cdots\right\}$; and we prove more-that $X$ is now countably compact. Given a sequence $\{x(m) \mid m=1,2, \cdots\}$ of points of $X$, we must produce a cluster point of it.

Let $i_{1}=$ first $n$, if any, for which infinitely many of the sets

$$
\mathrm{Cl}\left(x_{n}(m)\right), \quad m \in N=\{1,2, \cdots\}
$$

have nonempty intersection; say $p_{i_{1}} \in \bigcap\left\{\mathrm{Cl}\left(x_{i_{1}}(m)\right) \mid m \in M_{1}\right\}$, where $M_{1}$ is an infinite subset of $N$. Let $i_{2}=$ first $n>i_{1}$, if any, such that there exists an infinite $M_{2} \subset M_{1}$ with some $p_{i_{2}} \in \bigcap\left\{\mathrm{Cl}\left(x_{n}(m)\right) \mid m \in M_{2}\right\}$; and so on. We get a subset $I=\left\{i_{1}, i_{2}, \cdots\right\}$ of $N$, perhaps finite (or even empty), with $i_{1}<i_{2}<\cdots$. If $I$ is finite, the process terminates with some $i_{r}$; we put $M^{*}=M_{r}(=N$ if $r=0$, that is, if $I=\varnothing)$. If $I$ is infinite, let $m_{s}=s$ th member of $M_{s}(s=1,2, \cdots)$, and put $M^{*}=\left\{m_{s} \mid s \in N\right\}$. In either case, we have that $M^{*}$ is an infinite subset of $N$, and: (1) If $n \in I$, $p_{n} \in \mathrm{Cl}\left(x_{n}(m)\right)$ for all large enough $m \in M^{*}$. (2) If $n \notin I$, the system $\left\{\mathrm{Cl}\left(x_{n}(m)\right) \mid m \in M^{*}\right\}$ is point-finite. Now put $N-I=J$, and let $J=\left\{j_{1}, j_{2}, \cdots\right\}$ where $j_{1}<j_{2}<\cdots ; J$ may be finite (or empty-but if $J=\varnothing$ we are through). 
Write $F_{k}=\left\{x(m) \mid m \in M^{*}, m>k\right\}, \mathscr{F}_{0}=\left\{F_{k} \mid k=1,2, \cdots\right\} ; \mathscr{F}_{0}$ is a filter base. Because $X_{j_{1}}$ is $K$-compact (and therefore countably compact), $\pi_{j_{1}}\left(\mathscr{F}_{0}\right)$ has an adherent point $q_{j_{1}}$. Now $q_{j_{1}}$ is not a $P$-point. For, by (2), we have $q_{j_{1}} \in X_{j_{1}}$ - $\mathrm{Cl}\left(x_{j_{1}}(m)\right)$ for all $m>$ some $k$; hence, if $q_{j_{1}}$ were a $P$-point it would have a neighborhood disjoint from $\pi_{j_{1}}\left(F_{k}\right)$, giving a contradiction. We omit the rest of the proof, as it is essentially the same as the second half of Glicksberg's.

\section{Sequentially compact spaces.}

5.1. Definition. As usual, we call a space $X$ sequentially compact providing each sequence $\{x(n) \mid n \in N\}$, where $N=\{1,2, \cdots\}$, has a convergent subsequence $\left.{ }^{16}\right)$. As is well known, every sequentially compact space is countably compact and therefore feebly compact; in the converse direction, a countably compact space, in which each non- $P$ point has a countable base of neighborhoods, is easily seen to be sequentially compact. Thus the case $K=\aleph_{0}$ of Theorem 4.7 (but not Theorem 4.4) is included in the following theorem.

5.2. THEOREM. Every product of feebly compact spaces, of which all but one (at most) are sequentially compact, is feebly compact.

The proof is immediate from the three observations: (1) it is enough to prove this for products of countably many spaces, (2) every product of countably many sequentially compact spaces is sequentially compact, (3) the product of a sequentially compact space and a feebly compact space is feebly compact.

We now consider what can be said when all the factor spaces are sequentially compact. The product will in general (when there are uncountably many factors) not be sequentially compact, as the following example shows (5.3). It would be interesting to know whether every product of sequentially compact spaces must be countably compact. We shall see that this is so in two special cases $(5.5,5.8)$, but the general question remains open.

5.3. EXAMPLE. The space $2^{c}$ is not sequentially compact.

Here $2^{c}$ denotes the product of $c\left(=2^{\mathrm{N}_{0}}\right)$ spaces each consisting of 2 isolated points; it is, of course, compact. Let $S$ be the set of all infinite subsets of the set $N$ of positive integers, a set of cardinal $c$, and for each $s \in S$ choose disjoint infinite subsets $a_{s}, b_{s}$ with union $s$. Let $X_{s}$ be the space consisting of $a_{s}$ and $b_{s}$ (regarded as isolated points) as members; then $\prod\left\{X_{s} \mid s \in S\right\}$ is just $2^{c}$. We define a sequence $\{x(n) \mid n \in N\}$ of points of $2^{c}$ by specifying that the sth coordinate $x_{s}(n)$ is $a_{s}$ if $n \in a_{s}, b_{s}$ if $n \in b_{s}$. If this has a convergent subsequence $\{x(n) \mid n \in t\}$, then $t \in S$, and the $t$ th coordinate $x_{t}(n)$ takes both the values $a_{t}, b_{t}$ for arbitrarily large $n \in t$; the sequence $\left\{x_{t}(n) \mid n \in t\right\}$ thus does not converge, giving a contradiction.

5.4. Corollary. Assuming the continuum hypothesis, the product of an uncountable family of $T_{1}$ spaces, each having more than one point, is never sequentially compact.

(16) A sequence may converge to more than one limit. 
For it has $2^{c}$ as a closed subspace.

5.5. THEOREM. Every product of at most $\aleph_{1}$ sequentially compact spaces is countably compact.

It is enough to consider $X=\prod X_{a}$ where $a$ now runs over the set of all countable ordinals, and where each $X_{a}$ is sequentially compact. Given a sequence $\{x(n) \mid n \in N\}$ of points of $X$, we produce a cluster point. As in 5.3, let $S$ be the set of all infinite subsets of $N$. We construct, by transfinite induction over $a$, a set $s(a) \in S$ for each $a$, such that

(1) The sequence $\left\{x_{a}(n) \mid n \in s(a)\right\}$ converges to some $y_{a} \in X_{a}$.

(2) Whenever $b<a, s(a)-s(b)$ is finite.

For suppose this done for all $a^{\prime}<a$, and consider three cases.

(i) If $a=0$, we take $s(0)$ to be any member of $S$ for which $\left\{x_{0}(n) \mid n \in s(0)\right\}$ converges (in $X_{0}$ ).

(ii) If $a=b+1$, the sequence $\left\{x_{a}(n) \mid n \in s(b)\right\}$ (in $X_{a}$ ) has a convergent subsequence $\left\{x_{a}(n) \mid n \in t\right\}$ for some infinite $t \subset s(b)$; we take $s(a)=t$, noting that if $b^{\prime}<b$ then $s(a)-s\left(b^{\prime}\right) \subset s(b)-s\left(b^{\prime}\right)$ which is finite.

(iii) If $a$ is a limit ordinal, we take a sequence of ordinals $b_{1}<b_{2}<\cdots$ with $\sup b_{k}=a$. Take $n_{1} \in s\left(b_{1}\right)$ so large that, whenever $n>n_{1}$ and $n \in s\left(b_{2}\right)$ it follows that $n \in s\left(b_{1}\right)$; this is possible because, by assumption, $s\left(b_{2}\right)-s\left(b_{1}\right)$ is finite. Take $n_{k}$ recursively so that $n_{k} \in s\left(b_{k}\right), n_{k}>n_{k-1}$, and whenever $n>n_{k}$ and $n \in s\left(b_{k+1}\right)$ it follows that $n \in s\left(b_{k}\right)$. This defines a set $t=\left\{n_{k} \mid k \in N\right\} \in S$. The sequence $\left\{x_{a}(n) \mid n \in t\right\}$ has a convergent subsequence $\left\{x_{a}(n) \mid n \in s(a)\right\}$ for some infinite $s(a) \subset t$; we prove that $s(a)$ has the desired properties. Clearly (1) continues to hold, so we have only to verify (2). Suppose $b<a$; then, for large enough $k$, we have $b<b_{k}<a$. If $n \in s(a)$ and $n>n_{k}$, then $n \in t$ and so $n=n_{i}$ for some $i>k$; thus $n \in s\left(b_{i}\right)$ and $n>n_{i-1}$, so $n \in s\left(b_{i-1}\right)$; and by repeating this argument we obtain $n \in s\left(b_{k}\right)$. Thus $s(a)-s\left(b_{k}\right)$ consists only of numbers $\leqq n_{k}$, and is therefore finite. But $s\left(b_{k}\right)-s(b)$ is also finite; hence so is $s(a)-s(b)$, as required.

Thus we have, for each $a \in A$, a set $s(a) \in S$ such that (1) and (2) hold. The point $y=\prod\left\{y_{a} \mid a \in A\right\}$ (where $y_{a}$ is as in (1)) is easily seen to be a cluster point of the sequence $\{x(n) \mid n \in N\}$, and the theorem is proved.

In considering the general question as to the countable compactness of an arbitrary product $X=\prod X_{a}$ of (sequentially compact) spaces, it is evidently sufficient to restrict attention to the case in which each $X_{a}$ is separable (has a countable dense set). For, given a sequence $\{x(n) \mid n \in N\}$ in $X$, we may replace $X_{a}$ by the closure (in $X_{a}$ ) of the set $\left\{x_{a}(n) \mid n \in N\right\}$. Another reduction of the problem is given by the next theorem; it is enough to consider products of at most $2^{2^{c}}$ factors.

5.6. THEOREM. If $X=\prod X_{a}$, and every product of at most $2^{2^{c}}$ of the spaces $X_{a}$ is countably compact, then $X$ is countably compact. 
Given a sequence $\{x(n) \mid n \in N\}$ in $X$, we observe that (for each $a \in A) X_{a}$ is countably compact, so the sequence $\left\{x_{a}(n) \mid n \in N\right\}$ has a nonempty set $C_{a}$ of cluster points in $X_{a}$. For each $c_{a} \in C_{a}$, and for each neighborhood $U_{a}$ of $c_{a}$, consider the set $\left\{n \mid x_{a}(n) \in U_{a}\right\}$; as $U_{a}$ varies, these sets form a filter $F_{a}\left(c_{a}\right)$ on $N$. Let $\Phi_{a}$ denote the set $\left\{F_{a}\left(c_{a}\right) \mid c_{a} \in C_{a}\right\}$, and write $\left\{\Phi_{a} \mid a \in A\right\}=\Lambda$. There are only $2^{c}$ filters on $N$, so the cardinal of $\Lambda$ is at most $2^{2 c}$. For each $\lambda \in \Lambda$, put $A_{\lambda}=\left\{a \mid \Phi_{a}=\lambda\right\}$; the sets $A_{\lambda}$ are disjoint and nonempty, and their union is $A$.

Each $a \in A$ belongs to a unique $A_{\lambda}$, say for $\lambda=\mu(a)$. We select one element, denoted by $b(\lambda)$, in each $A_{\lambda}$. (Thus $\mu(b(\lambda))=\lambda$.) Consider the product $Y=\prod\left\{X_{b(\lambda)} \mid \lambda \in \Lambda\right\}$, and let $\pi$ denote the projection of $X$ onto $Y$. By hypothesis, $Y$ is countably compact, so the sequence $\{\pi(x(n)) \mid n \in N\}$ has a cluster point $y$ in $Y$. We define a cluster point $z$ of the given sequence in $X$ as follows. For each $a \in A, y_{b(\mu(a))} \in C_{b(\mu(a))}$; the filter $F_{b(\mu(a))}\left(y_{b(\mu(a))}\right)$ is thus defined, and belongs to $\Phi_{b(\mu(a))}$. But $b(\mu(a))$ and $a$ belong to the same $A_{\lambda}$, so $\Phi_{b(\mu(a))}=\Phi_{a}$. We may therefore choose $z_{a} \in C_{a}$ so that $F_{a}\left(z_{a}\right)=F_{b(\mu(a))}\left(y_{b(\mu(a))}\right)$. This defines $z=\prod\left\{z_{a} \mid a \in A\right\} \in X$; we verify that $z$ is a cluster point of $\{x(n) \mid n \in N\}$.

Given a neighborhood $U$ of $z$ in $X, U$ contains one of the form $V=\prod V_{a}$, where $V_{a}$ is a neighborhood of $z_{a}$ in $X_{a}$, and where $V_{a}=X_{a}$ for all $a \in A-E$, where $E$ is finite. The set $\mu(E)$ is, of course, finite too. For each $\lambda \in \mu(E)$, we consider the finite set $E \cap A_{\lambda}$; for each $a \in E \cap A_{\lambda}$ we have $\mu(a)=\lambda$ and

$$
\left\{n \mid x_{a}(n) \in V_{a}\right\} \in F_{a}\left(z_{a}\right)=F_{b(\mu(a))}\left(y_{b(\mu(a))}\right)=F_{b(\lambda)}\left(y_{b(\lambda)}\right) .
$$

Since this is a filter, the intersection $\left\{n \mid x_{a}(n) \in V_{a}\right.$ for all $\left.a \in E \cap A_{\lambda}\right\}$ is also a member of $F_{b(\lambda)}\left(y_{b(\lambda)}\right)$. That is, there exists a neighborhood $W_{b(\lambda)}$ of $y_{b(\lambda)}$ such that

(1) $x_{b(\lambda)}(n) \in W_{b(\lambda)}$ if and only if $x_{a}(n) \in V_{a}$ for all $a \in E \cap A_{\lambda}$.

We write $W_{b(\lambda)}=X_{b(\lambda)}$ if $\lambda \notin \mu(E)$. The set $W=\prod\left\{W_{b(\lambda)} \mid \lambda \in \Lambda\right\}$ is then a neighborhood of $y$ in $Y$, and it therefore contains $\pi(x(n))$ for arbitrarily large $n$. From (1) it follows that $V$ (and so $U$ ) contains $x(n)$ for arbitrarily large $n$, and the proof is complete.

5.7. CoROllary. If $X=\prod X_{a}$, where each $X_{a}$ is first countable, and if every product of at most $2^{c}$ of the spaces $X_{a}$ is countably compact, then $X$ is countably compact.

For we can here restrict attention to countably-based filters on $N$ in the preceding argument, and there are only $c$ of these. (Note that the hypotheses imply that each $X_{a}$ is sequentially compact here.)

5.8. THEOREM. Let $Z$ be any sequentially compact space of at most $c$ points. Then, assuming the continuum hypothesis, $Z^{K}$ is countably compact, for every cardinal $K\left({ }^{17}\right)$.

(17) The hypotheses apply whenever $Z$ is sequentially compact, separable and Hausdorff. 
Here $Z^{K}$ denotes $\prod\left\{X_{a} \mid a \in A\right\}$ where each $X_{a}=Z$ and $A$ has cardinal $K$. Because of Theorem 5.5, we may assume $K \geqq \aleph_{1}=c$.

Given a sequence $\{x(n) \mid n \in N\}$ in $Z^{K}$, we must as usual produce a cluster point. Let $\mathscr{S}$ be the family of all sequences (of points of $Z$ ) (that is, of maps $N \rightarrow Z$ ) of the form $\left\{x_{a}(n) \mid n \in N\right\}$ for at least one $a \in A$; then $\mathscr{S}$ has cardinal $\leqq c^{\text {}_{0}}=c$, and we write $\mathscr{S}=\left\{S_{\lambda} \mid \lambda \in \Lambda\right\}$, where $\Lambda$ is an index set of cardinal $\leqq c$. Let $A_{\lambda}=\left\{a \mid\left\{x_{a}(n) \mid n \in N\right\}=S_{\lambda}\right\}$; the sets $A_{\lambda}$ form a partition of $A$. From each $A_{\lambda}$ select one element $b(\lambda)$, and put $Y=\prod\left\{X_{b(\lambda)} \mid \lambda \in \Lambda\right\}$. Let $\pi$ denote the projection of $Z^{K}$ onto $Y$. By Theorem 5.5, $Y$ is countably compact, so the sequence $\{\pi(x(n)) \mid n \in N\}$ has a cluster point $y$. For each $a \in A$ define $\mu(a)=$ the value of $\lambda$ for which $a \in A_{\lambda}$, and take $p_{a}=y_{\mu(a)}$. By an argument similar to that in 5.6 one verifies that $p=\prod\left\{p_{a} \mid a \in A\right\}$ is a cluster point of the given sequence $\{x(n) \mid n \in N\}$, as required.

\section{REFERENCES}

1. P. Alexandroff and H. Hopf, Topologie I, Die Grundlehren der mathematischen Wissenschaften in Einzeldarstellungen, 45, Springer, Berlin, 1935.

2. R. W. Bagley, E. H. Connell and J. D. McKnight, Jr., On properties characterizing pseudocompact spaces, Proc. Amer. Math. Soc. 9 (1958), 500-506.

3. B. Banaschewski, über zwei Extremaleigenschaften topologischer Räume, Math. Nachr. 13 (1955), 141-150.

4. - Uber Hausdorfssch-minimale Erweiterung von Räumen, Arch. Math. 12 (1961), 355-365.

5. M. P. Berri, Minimal topological spaces, Trans. Amer. Math. Soc. 108 (1963), 97-105.

6. _- Categories of certain minimal topological spaces, J. Austral. Math. Soc. 4 (1964), 78-82.

7. M. P. Berri and R. H. Sorgenfrey, Minimal regular spaces, Proc. Amer. Math. Soc. 14 (1963), 454-458.

8. N. Bourbaki, Espaces minimaux et espaces complètement séparés, C. R. Acad. Sci. Paris. 205 (1941), 215-218. 1965.

9. ——, Topologie générale, 4th ed., Actualités Sci. Ind. No. 1142, Hermann, Paris,

10. C. Chevalley and O. Frink, Jr., Bicompactness of Cartesian products, Bull. Amer. Math. Soc. 47 (1941), 612-614.

11. I. Glicksberg, Stone-Čech compactifications of products, Trans. Amer. Math. Soc. 90 (1959), 369-382.

12. D. Hall and G. L. Spencer II, Elementary topology, Wiley, New York, 1955.

13. E. Hewitt, On two problems of Urysohn, Ann. of Math. (2) 47 (1946), 503-509.

14. H. Herrlich, $T_{v}$-Abgeschlossenheit und $T_{v}$-Minimalität, Math. Z. 88 (1965), 285-294.

15. S. Ikenaga, Product of minimal topological spaces, Proc. Japan Acad. 40 (1964), 329-331.

16. J. L. Kelley, General topology, Van Nostrand, New York, 1955.

17. J. Novak, On the Cartesian product of two compact spaces, Fund. Math. 40 (1953), 106-112.

18. F. Obreanu, Filtre deschise, Acad. Repub. Pop. Române Bul. Şti. Mat. Fiz. Chim. 2 (1950), 1-5.

19. - Spatii absolut inchise, ibid, (1950), 21-25. 
20. - Spatii separate minimale, An. Acad. Repub. 'Pop. Române Sec. Mat. Şti. Fiz. Chim. Ser. A 3 (1950), 325-349.

21. —_ Asupra unei probleme a lui Alexandrov şi Urysohn, ibid. (1950), 101-108.

22. A. H. Stone, Hereditarily compact spaces, Amer. J. Math. 82 (1960,) 900-916.

23. M. H. Stone, Applications of the theory of Boolean rings to general topology, Trans. Amer. Math. Soc. 41 (1937), 375-481.

24. P. Urysohn, Über die Mächtigkeit der zusammenhängenden Mengen, Math. Ann. 94 (1925), 266-295.

TULANE UNIVERSTTY,

New OrLeans, Louisiana

UNIVERSITY OF ROCHESTER,

ROCHESTER, NEW YORK 